\title{
Frequency of the first feature in action sequences influences feature binding
}

\author{
Paul S. Mattson • Lisa R. Fournier • \\ Lawrence P. Behmer Jr.
}

Published online: 10 July 2012

(C) Psychonomic Society, Inc. 2012

\begin{abstract}
We investigated whether binding among perception and action feature codes is a preliminary step toward creating a more durable memory trace of an action event. If so, increasing the frequency of a particular event (e.g., a stimulus requiring a movement with the left or right hand in an up or down direction) should increase the strength and speed of feature binding for this event. The results from two experiments, using a partial-repetition paradigm, confirmed that feature binding increased in strength and/or occurred earlier for a high-frequency (e.g., left hand moving up) than for a low-frequency (e.g., right hand moving down) event. Moreover, increasing the frequency of the first-specified feature in the action sequence alone (e.g., "left" hand) increased the strength and/or speed of action feature binding (e.g., between the "left" hand and movement in an "up" or "down" direction). The latter finding suggests an update to the theory of event coding, as not all features in the action sequence equally determine binding strength. We conclude that action planning involves serial binding of features in the order of action feature execution (i.e., associations among features are not bidirectional but are directional), which can lead to a more durable memory trace. This is consistent with physiological evidence suggesting that serial order is preserved in an action plan executed from memory and that the first feature in the action sequence may be critical in preserving this serial order.
\end{abstract}

Keywords Perception and action - Repetition effects . Priming $\cdot$ Action planning

P. S. Mattson • L. R. Fournier $(\bowtie) \cdot$ L. P. Behmer Jr. Psychology Department, Washington State University, Pullman, WA 99164-4820, USA

e-mail: lfournier@wsu.edu
The primate brain codes both perceptual features (e.g., the shape, color, and movement of an object) and action features (e.g., a limb and the movement direction of the limb) in a distributed fashion, with each feature represented by a distinct cell assembly in different areas of the brain (Georgopoulos, 1990; Kalaska \& Hyde, 1985; Riehle \& Requin, 1989; Treisman, 1996). Because people often carry out more than one task at a time, the stimuli and responses that belong to the same task (event) may be temporarily linked (or bound) to some degree to keep the perception and actions relevant to one event separate from those relevant to another (Hommel, 2004, 2005). The theory of event coding (TEC) assumes that feature codes - meaning-based representations of perceptual stimuli and intended action operations (e.g., the code "left" for a left-moving stimulus or for a left-hand response) - are activated and are temporarily bound so as to represent a single event (Fournier, Wiediger, McMeans, Mattson, Kirkwood, \& Herzog, 2010; Hommel, Müsseler, Aschersleben, \& Prinz, 2001; Müsseler \& Hommel, 1997; Wiediger \& Fournier, 2008; Wühr, 2006). Temporary bindings among the perception and action feature codes associated with the same event are referred to as an "event file" or action plan (Hommel et al., 2001). Although event files (and, hence, action plans) are assumed to be temporary representational structures, it is possible that the binding of perception and action feature codes is a preliminary step toward creating a long-term representational structure (Colzato, Raffone, \& Hommel, 2006). Consistent with this assumption, some evidence indicates that perception and action information can be represented as an integrated structure in long-term memory. For example, passive observations of tools (e.g., scissors) can evoke neuronal activation in different cortical motor areas associated with tool use (Chao \& Martin, 2000; Grafton, Fadiga, Arbib, \& Rizzolatti, 1997; Martin, Wiggs, Ungerleider, \& Haxby, 1996). 
If binding among perception and action feature codes is a preliminary step toward creating a more durable memory trace of the action event, then increasing the frequency of a particular event should increase the strength and speed of feature binding of this event prior to forming a long-term memory structure (Colzato, Raffone, \& Hommel, 2006). In this study, we examined whether increasing an event's frequency can improve the strength and speed of binding among multiple action feature codes (e.g., the perception and action feature codes "left," representing a left-hand action, and "up," representing the action of moving upward).

One paradigm used to show that feature codes associated with the same event are temporarily bound together to form a single event file (or action plan) was developed by Stoet and Hommel (1999) and modified by Wiediger and Fournier (2008). In the Wiediger and Fournier study, two different visual events were presented sequentially. Participants were instructed to plan and maintain a sequence of keypresses (lateral movements "up" or "down" on a keypad) with either their right or left hand, based on the stimulus identity of the first event (Event A). While maintaining the action plan for Event A (e.g., "left hand move up"), a second visual event appeared (Event B). An immediate, single keypress response with either the right or the left hand was required (with no lateral movement on a keypad), based on the stimulus identity of Event B. After executing a speeded response to Event B (e.g., a "left" hand press), the planned action to Event A (e.g., "left hand move up") was executed. The results showed that the response to Event B was longer in duration when it required the same hand as Event A (e.g., left), as opposed to a different hand (e.g., right). That is, maintaining an action plan in memory for later execution can delay the execution of another (new) action if there is partial feature overlap between the two action events (e.g., both actions require the same hand code, "left," and different movement codes, "up" vs. no lateral movement), as opposed to no feature overlap (e.g., both actions require different hand codes, "left" vs. "right," and different movement codes, "up" vs. no lateral movement). This delay in executing the new action plan due to partial feature overlap, where some but not all action features overlap with the action plan maintained in memory, is referred to as compatibility interference or a partial-repetition cost (PRC).

Consistent with the theory of event coding (Hommel, 2004, 2005), it is assumed that PRCs occur because the feature codes for Event A (e.g., "left" for the left hand and "up" for moving the hand upward) are bound together, and hence these feature codes (e.g., "left" and "up") can no longer be activated independently. That is, binding leads to an associated connection between the respective feature codes, such that activating one code will spread activation to the other codes it is associated with. In cases in which there is partial overlap among the action codes between two events, interference is expected. For example, if a feature code from Event B (e.g., "left") matches one of the Event A codes, this Event B code (e.g., "left") will activate the Event A code as well as the other Event A code that it is bound to (e.g., "up"). As a result, time will be required in order to override the activation of the code (e.g., "up") that is irrelevant to the Event B response, so as to correctly select the Event B response. The time necessary to override the code activation irrelevant to Event B (e.g., "up") will delay the response to Event B (Hommel, 2004, 2005; Mattson \& Fournier, 2008). ${ }^{1}$ See Fig. 1. In cases in which there is no action code overlap between the two events, no interference is expected. For example, if no codes from Event B (e.g., "left") match those for Event A (e.g., "right" and "up"), no Event A codes will be activated, and hence no extra time will be required to override any irrelevant Event A codes before selecting the Event B response. Again, see Fig. 1. Finally, in cases of complete feature code overlap between the two events (i.e., the events are identical), facilitation is expected rather than interference. Here, no irrelevant codes will be activated when selecting the response to Event B, and the Event A codes will prime those relevant to Event B, leading to a speed-up in Event B responses.

A different outcome is expected when there is partial feature code overlap between Event B and Event A if the feature codes for Event A are not yet bound (Hommel, 2004, 2005). In this case, partial-repetition facilitation (PRF) should result, as opposed to a cost. This outcome is expected because there is no reliable associated connection between Event A feature codes, and activating one Event A code will not spread activation to the other Event A codes. As a result, each Event A code can be activated independently, and each Event A code can independently activate its associated response as well. Hence, no interference should occur when a feature code from Event B (e.g., "left") matches one of the feature codes from Event A (e.g., "left" among the unbound codes "left" and "up"); instead, the matching code from Event A should prime activation of the Event B code and facilitate the Event B response.

Importantly, the predicted outcome for partial feature overlap between two events differs depending on whether or not the Event A feature codes are bound. In contrast, outcomes for cases of complete feature overlap or no feature overlap between Event A and Event B are not dependent on whether or not Event A feature codes are bound; the effect

\footnotetext{
${ }^{1}$ Both a code confusion and a code occupation account (Hommel et al., 2001; Stoet \& Hommel, 1999) have been used to explain PRCs, although the code confusion idea we will describe in the text has been put forth in more recent publications (e.g., Hommel, 2004, 2005; Mattson \& Fournier, 2008). Importantly, the performance predictions related to feature binding are the same in both accounts (at least in this study).
} 


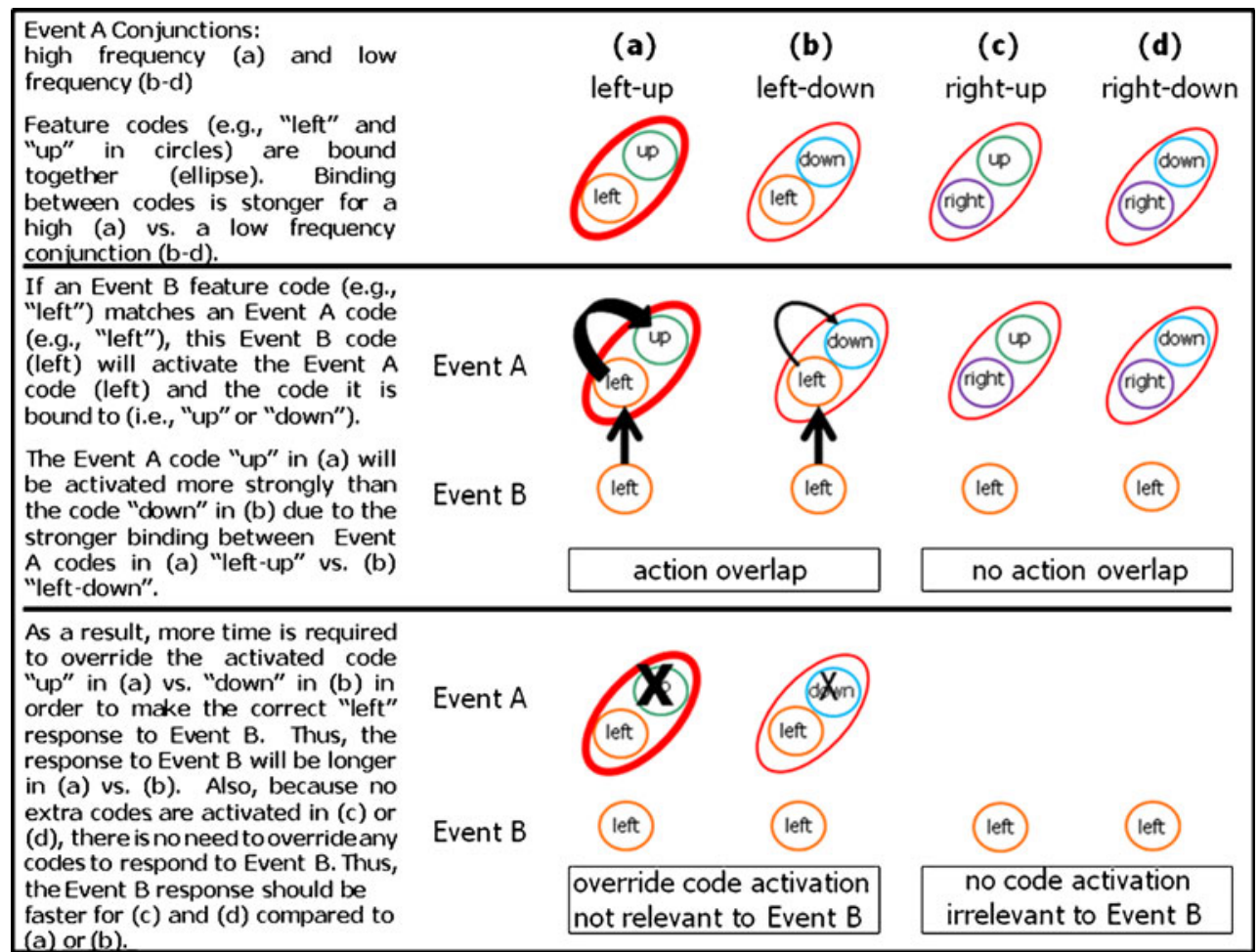

Fig. 1 Predictions consistent with the theory of event coding. (Top) The four possible feature conjunctions (hand + movement) for Event A (a-d). Individual feature codes (representing left/right hand and up/ down movement) for each conjunction are represented by the small circles. The ellipses represent the binding of these feature codes into a coherent event representation: The thick ellipse (a) represents the highfrequency conjunction (where binding between codes should be stronger), and the thin ellipses (b-d) represent each of the low-frequency conjunctions (where binding strength should be relatively weaker). (Middle) The bound Event A conjunctions along with the Event B feature code (e.g., "left" hand), represented by the small circle (note: the Event B feature code representing the right hand is not shown). Overlap between the "hand" feature codes (e.g., "left") for Events B and $\mathrm{A}$ is depicted in columns (a) and (b), and no feature overlap between the "hand" feature codes for Event B and A is depicted in columns (c) and (d). According to the TEC, a partial-repetition cost should be found if the Event B action feature code(s) partly overlap (action overlap) with any bound features representing Event A. That is, if an Event B feature code (e.g., "left") matches one of the Event A

on the Event B response should be the same-facilitation, in the case of complete overlap, and neither facilitation nor a cost, in the case of no overlap. Thus, Event B performance (a cost or facilitation) in the case of partial rather than no feature overlap can provide information as to whether or not the features of Event A are bound. If there is a PRC, the feature codes are bound; if there is a PRF, the feature codes are not bound. Furthermore, the relative size of a PRC should reflect the relative binding strength among action feature codes, and the relative size of the PRF should reflect the relative degree of independence among feature codes.

According to the TEC, which feature codes within an event become bound together into an event file or action plan is determined by the activation level of the individual codes (e.g., "left"), the Event B feature code will activate this Event A code (see vertical arrows) and the other code(s) that it is bound to (see arrows connecting the Event A code "left," activated by Event B, to the other Event A codes "up" and "down" with which it is bound). The Event A code will be activated more strongly (indicated by the thicker arrow) at (a) than at (b) because this Event A code is more strongly bound to the Event A code activated by Event B. There is no feature code overlap between Events A and B at (c) and (d), and hence no Event A codes should be activated. (Bottom) The irrelevant-code activations corresponding to Event A (e.g., "up" and "down") in (a) and (b) must be inhibited (depicted by the $\mathrm{X}$ ) in order for the participant to respond correctly to Event B. Also, more time (indicated by the thicker $\mathrm{X}$ ) is required to inhibit the irrelevant code in (a) as compared to the one at (b), because the irrelevant code is more strongly activated in (a) than in (b). Hence, the delay, or partial-repetition cost, should be larger in (a) than in (b). No irrelevant codes need to be inhibited in (c) and (d), where there is no feature code overlap, and hence no delays are expected when responding to Event B

feature codes. Thus, binding is not instantaneous; there are two phases to the binding process (Colzato, Raffone, \& Hommel, 2006; Hommel et al., 2001). In the first phase, the feature codes associated with an event are activated, and goal-relevant features will have a higher level of activation than will goal-irrelevant features. In the second phase, feature codes with relatively high activations are more likely to be integrated into a temporary event file (perhaps due to enhancing or synchronizing the firing patterns of neural units representing features of the same event; Desimone \& Duncan, 1995; Engel \& Singer, 2001; Singer, 1994). In addition, the higher the activation strength of the feature code(s), the greater the binding strength among those feature codes. From these assumptions, increasing the activation 
level of a feature code should increase the strength of the binding that that feature code will form. One way to increase the activation level of a feature code, and in turn to increase binding strength, may be to make specific goal-relevant features occur more frequently (e.g., Colzato, Raffone, \& Hommel, 2006).

Colzato, Raffone, and Hommel (2006) examined whether the frequency of an event would affect its feature-binding strength. They failed to find an increase in binding strength (larger PRCs) among visual features when specific conjunctions of visual features appeared more frequently than others. However, it is unclear whether the same would hold true for a conjunction of action features associated with a specific visual event (but see Colzato, Warrens, \& Hommel, 2006). Also, Colzato et al.'s failure to obtain frequency effects on binding strength may have been due to a lack of sensitivity in their frequency manipulation (which occurred only during practice trials) and the paradigm they used to assess PRCs; only small PRCs were obtained, and only in early trials.

It is well known that frequent pairing of a stimulus with a response increases the association strength between the two, and as a result, presentation of the stimulus can prime (activate) its associated response (e.g., Eriksen \& Eriksen, 1974; Forster, Liberman, \& Friedman, 2008; Terry, 2003; Voss, 1972). Also, associative learning in humans is accompanied by neural synchronization between brain areas representing the to-be-associated stimuli (e.g., Miltner, Braun, Arnold, Witte, \& Taub, 1999). Thus, in contrast to the findings by Colzato, Raffone, and Hommel (2006), repetition of an action event may increase the activation level and the association strength (i.e., the binding) among the taskrelevant feature codes of this event. If so, we would expect larger PRCs when the action maintained in working memory is a high-frequency event (i.e., a high-frequency conjunction) as opposed to a low-frequency event (i.e., a lowfrequency conjunction).

In the present study, we investigated whether binding strength and/or the onset of the binding process among action feature codes (i.e., "left" or "right" representing the action hand and "up" or "down" representing the hand movement) are influenced by the frequency of the specific hand movement event (i.e., by the conjunction). Our two experiments were based on a delayed response task similar to that of Wiediger and Fournier (2008). The first action event (Event A) varied in frequency, and this event was maintained in memory while executing a response to the second action event (Event B). For Experiment 1, we examined whether a high-frequency event (high-frequency conjunction) has stronger feature code bindings than a low-frequency event (low-frequency conjunction). In Experiment 2 , we examined whether the binding of feature codes can occur earlier for a high- than for a low-frequency conjunction. If a high-frequency conjunction leads to stronger and/or earlier feature binding, this would suggest that binding among feature codes can be a preliminary step for creating a more durable memory trace. If repetition of a high-frequency conjunction does not lead to stronger and/or earlier feature binding, this would suggest that binding among feature codes is temporary and may serve only to keep the perceptions and actions corresponding to one event separate from those corresponding to another.

\section{Experiment 1}

In Experiment 1, we examined whether increased event frequency of a specific hand (left or right) and movement (up or down) conjunction can increase the binding strength among hand and movement action features. Our participants saw a visual event (Event A) that required them to plan an action (e.g., "left hand move up") and to maintain this action plan in working memory. Then, they saw a second visual event (Event B) that either shared the same response hand (e.g., left) with Event A (action overlap) or did not (e.g., right; no action overlap). When the two action plans shared the same "hand" feature code in common (e.g., "left" for the left hand), a PRC was expected (i.e., elevated response times [RTs] and/or errors for Event B responses). Event A frequency was also manipulated. One instance of Event A (a specific conjunction of hand [left or right] and movement [up or down] features; e.g., "left-up") appeared more frequently than the other instances (e.g., "leftdown," "right-up," and "right-down"). If increases in Event A frequency increase binding strength among the Event A action features associated with hand and movement, the size of the PRC should be larger when Event $\mathrm{A}$ is a high-frequency rather than a low-frequency conjunction (see Fig. 1).

Method

\section{Participants}

A group of 47 undergraduates (18-40 years old) from Washington State University participated in this experiment, approved by the Institutional Review Board (IRB). They received optional extra credit in their psychology courses. Participants had at least 20/60 visual acuity and correctly identified the colors red/green on a Snellen chart. Six of the participants were excluded for violating the task instructions (i.e., moved fingers or vocalized responses while planning the action for Event A), and one participant was excluded for failing to reach the $80 \%$ accuracy criterion during practice. The data were analyzed for the remaining 40 participants. 


\section{Apparatus}

The stimuli appeared on a 17-in. CRT monitor. Two keypads were located on a table in front of the participant $(6 \mathrm{~cm}$ in front of the CRT and $10.5 \mathrm{~cm}$ from the table edge), with one keypad $11 \mathrm{~cm}$ to the left and the other $11 \mathrm{~cm}$ to the right of the participant's body midline. The keypads recorded responses made with the index fingers: Left-hand responses were executed on the left keypad, and right-hand responses were executed on the right keypad. Each keypad had three keys $(1 \times 1 \mathrm{~cm}$ in size, separated by $0.2 \mathrm{~cm})$ oriented vertically. The key in the middle of each keypad was the home key. Participants rested their index fingers on the left and right home keys before and during each trial (see Fig. 2). The participant's hands and the keypads were visible if he or she looked down. E-Prime software (version 1.2; Psychology Software Tools, Inc., Sharpsburg, PA) was used for stimulus generation and presentation, as well as for data collection.

\section{Stimuli and responses}

The stimuli appeared on a black background. A white fixation cross (approximately $0.70^{\circ}$ of visual angle) appeared at screen center before and during each trial.

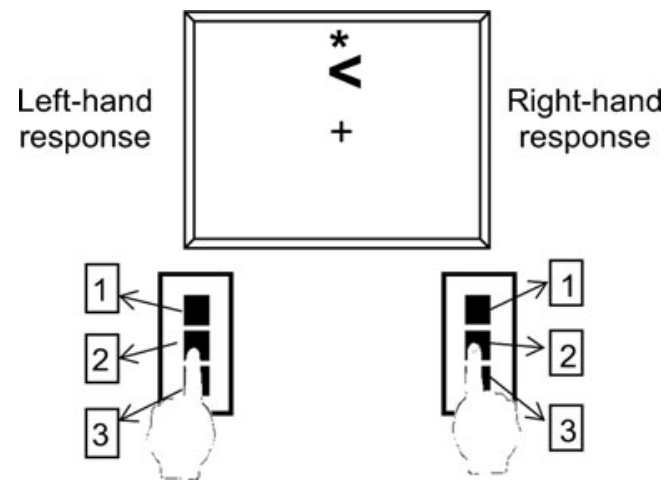

Fig. 2 A diagram of the response apparatus used to respond to Events $\mathrm{A}$ and $\mathrm{B}$ in Experiments 1 and 2. The lines pointing to the numbered boxes and the numbered boxes were not part of the apparatus; they are presented here to show the keypress sequence required for the different stimuli assigned to Event A. Event A consisted of an arrowhead $(<$ or $>$ ) and an asterisk $(*)$ that appeared above or below the arrowhead. The arrowhead direction indicated which hand (left or right, respectively) was required to execute a response. The asterisk above the arrowhead indicated a keypress sequence of 2-1-2, and the asterisk below the arrowhead indicated a keypress sequence of 2-3-2. For example: < with an asterisk above it (leftup response) required the left hand to press the key sequence 2$1-2$ on the left keypad; < with an asterisk below it (left-down response) required the left hand to press the key sequence 2-3-2 on the left keypad; > with an asterisk above it (right-up response) required the right hand to press the key sequence $2-1-2$ on the right keypad; and $>$ with an asterisk below it (right-down response) required the right hand to press the key sequence 2-3-2 on the right keypad
Event $A$ Event A was a white arrowhead (subtending $0.63^{\circ}$ of visual angle) and an asterisk (subtending $0.53^{\circ}$ of visual angle). The arrowhead pointed to the left or the right (" $<$ " or ">") and was centered $2^{\circ}$ of visual angle above the fixation cross. The asterisk was centered $0.74^{\circ}$ of visual angle above or below the arrowhead. The arrowhead direction (left or right) indicated the response hand (left or right, respectively). The asterisk location indicated the movement direction of the index finger from the center key (home key) on the keypad. The asterisk above the arrowhead indicated an "up" response (to press the key above the home key, toward the CRT), and the asterisk below the arrowhead indicated a "down" response (to press the key below the home key, toward the participant's body). Thus, the four different action plans varied in terms of response hand and movement direction (left-up, left-down, right-up, and right-down) and corresponded to the four different stimuli (left arrowhead with asterisk above, left arrowhead with asterisk below, right arrowhead with asterisk above, and right arrowhead with asterisk below, respectively). All of the responses began and ended by pressing the appropriate home key with the index finger. Figure 2 shows the different combinations of keypress responses for the four different action events.

Event $B$ Event B was a red or green pound sign (subtending $0.67^{\circ}$ of visual angle) centered $1.5^{\circ}$ of visual angle below the fixation cross. Participants made a two-choice, speeded response based on the color. Half of the participants pressed the left home key twice with their left hand when the pound sign was green and the right home key twice with their right hand when the pound sign was red; the other half had the opposite stimulus-response assignment.

\section{Procedure}

The participants were instructed to keep their left and right index fingers on the home keys of the left and right keypads, respectively, before and after responding to the different stimulus events. The trial sequence of events is presented in Fig. 3 and described below.

At the start of each trial, an initiation screen appeared with a fixation cross and a message that read "press the home keys to continue." When the home keys were pressed simultaneously, a fixation cross appeared for 1,000 ms. Afterward, Event A appeared above the fixation cross for $2,000 \mathrm{~ms}$, followed by the fixation cross alone for 1,250 ms. During this time, participants planned their response to Event A. Event B then appeared below the fixation cross for $250 \mathrm{~ms}$, and participants responded as quickly and as accurately as possible to Event B. A blank screen followed Event B and remained for 4,750 ms or until a response was detected. The Event B RT was calculated from 
Fig. 3 The sequence of trial events for Experiments 1 and 2

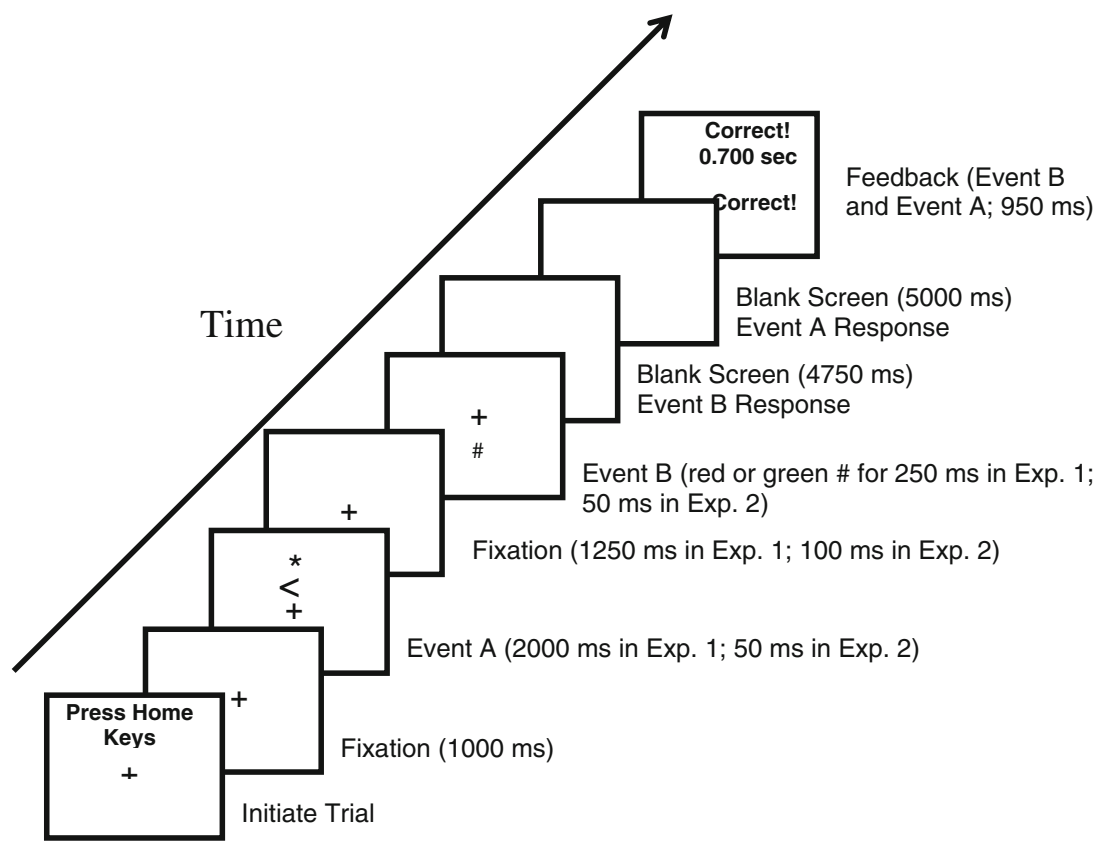

the onset of Event B to the first keypress detected. After responding to Event B, participants had 5,000 ms to execute the planned response to Event A. Participants were instructed to emphasize accuracy, not speed, when responding to Event A. Feedback indicating the Event B RT, Event B accuracy, and Event A accuracy was then presented for $950 \mathrm{~ms}$. Afterward, the initiation screen appeared, and participants started a new trial (when ready) by pressing the home keys simultaneously. The trial sequence then repeated.

Participants were instructed not to execute the planned response (or any part of the planned response) to Event A until after they had perceived and responded to Event B. In addition, they were told not to move any fingers, tense any muscles, or use any other external cues to help them remember the planned response sequence to Event A-they were instructed to hold the action sequence for Event A in memory.

To determine whether event (conjunction) frequency influences binding strength, two factors were manipulated. First, the action overlap between Event B and Event A was manipulated: The hand required to execute the correct action plan for Event B and Event A was either the same (action overlap) or different (no overlap). The action overlap conditions (overlap or no overlap) occurred in a random order with an equal probability of occurrence in each block of trials. The increase in Event B RTs and error rates in the action overlap relative to the no-overlap condition indicated a PRC. Second, the conjunction frequency of action features (hand and movement direction) mapped to Event A was manipulated. One of the four action feature conjunctions assigned to Event A (left-up, left-down, right-up, and right-down) occurred three times more often than each of the other three action feature conjunctions. The high- frequency conjunction assigned to Event A was balanced across participants, and each of the four hand movement conjunctions (represented by the arrowhead-and-asterisk stimulus combinations) served as the high-frequency conjunction equally often across participants. The higher frequency for one specific Event A conjunction (e.g., left-up) was intended to increase the binding strength among action features (e.g., "left-up") for that particular instance of Event A. Figure 1 illustrates the conjunction frequency manipulation and action overlap conditions.

In one 90- to 100-min session, participants completed one block of 16 practice trials and 36 blocks of 12 experimental trials. The practice trials (with no stimulus frequency manipulation) had an $80 \%$ accuracy criterion to continue the experiment; one participant failed to meet this criterion. In a block of 12 experimental trials, the high-frequency conjunction for Event A (e.g., left-up) was presented six times (three times in the action overlap and three times in the no-action-overlap condition), while each of the other three, low-frequency Event A conjunctions was presented twice (once in the action overlap and once in the no-actionoverlap condition). Participants took breaks after each threeblock sequence. To observe changes in performance with practice without drastically deceasing the stability in performance means, trials were grouped into early (Blocks 1-9), mid-early (Blocks 10-18), mid-late (Blocks 19-27), and late (Blocks 28-36) block groups.

After completing the experiment, participants answered questions concerning the strategies they used. Participants who reported or were observed moving fingers, tensing muscles on one side of the body, or using external cues (e.g., vocalizing the response out loud) when planning an action to Event A violated the explicit task instructions and 
were excluded from the analyses. This occurred for six participants. Consistent with hierarchical models of action planning (e.g., Rosenbaum, 1980; Rosenbaum, Inhoff, \& Gordon, 1984) and with evidence that the hand is prepared before the specific right- and left-hand fingerpress responses (Miller, 1982), all participants reported that they represented the action plan for Event A as "hand selection (left or right) followed by finger movement (up or down)."

Results and discussion

\section{Event A conjunction frequency}

A $2 \times 2 \times 4$ repeated measures analysis of variance (ANOVA) with the factors Action Overlap (overlap or no overlap), Event A Conjunction Frequency (high or low), and Block Group (early, mid-early, mid-late, or late) was conducted separately on the percentages of errors for Event A, on correct RTs for Event B, and on percentages of errors for Event B. The correct-RT and error analyses for Event B were restricted to trials in which the responses to Event A were correct. Figure 4 shows the mean correct RTs and percentages of errors for Event B, as a function of action overlap and Event A conjunction frequency (collapsed over block groups). As is evident in Fig. 4, PRCs were obtained that were greater in magnitude when Event A was the highfrequency conjunction.

Event $A$ accuracy The average error rate for Event A was $9.04 \%$. No significant effects were found ( $p$ s $>.05)$.

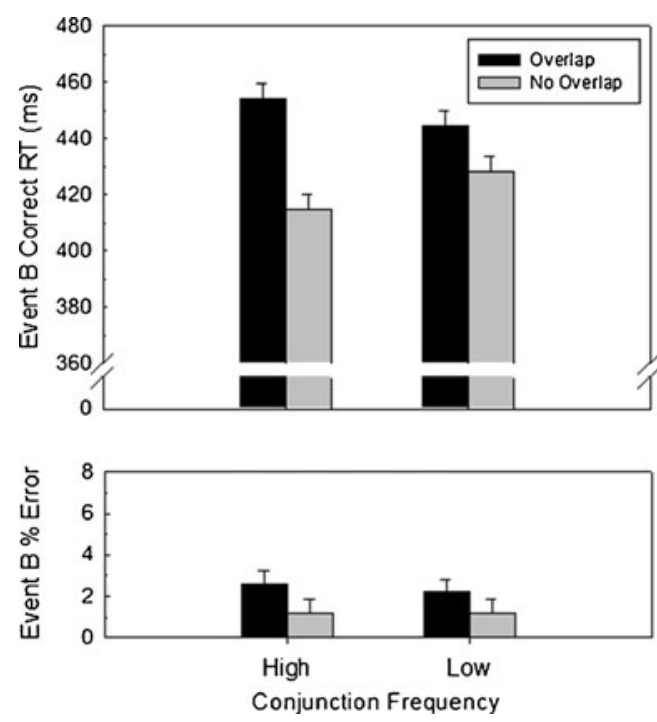

Fig. 4 Experiment 1: Event B correct RTs (upper panel) and percentages of errors (lower panel) for the action overlap and no-actionoverlap conditions when Event A was a high- or low-frequency conjunction. Each error bar represents one within-subjects standard error of the mean
Event $B$ RTs We found a significant effect of block group $\left[F(3,117)=30.37, p<.001, \eta_{\mathrm{p}}{ }^{2}=.44\right]$ showing that RTs decreased across blocks, consistent with a practice effect. Moreover, there was a significant effect of action overlap $\left[F(1,39)=32.89, p<.001, \eta_{\mathrm{p}}{ }^{2}=.46\right]$ and a significant interaction between action overlap and Event A conjunction frequency $\left[F(1,39)=4.63, p=.037, \eta_{\mathrm{p}}^{2}=.11\right]$. Planned comparisons showed that RTs were greater in the action overlap than in the no-overlap condition when Event A was either a high-frequency $(p<.001)$ or a low-frequency $(p<.01)$ conjunction. Also, the difference between the action overlap and no-overlap conditions was greater when Event A was the high-frequency conjunction $(M=39 \mathrm{~ms})$ rather than the lowfrequency conjunction $(M=16 \mathrm{~ms}), p=.037$. Thus, consistent with predictions, a PRC was obtained for high- and lowfrequency conjunctions, and the size of the PRC was larger for the high-frequency conjunction. See Fig. 4.

Event B accuracy The mean error rate was $1.8 \%$. Only a significant effect of action overlap $[F(1,39)=14.85$, $\left.p<.001, \eta_{\mathrm{p}}{ }^{2}=.28\right]$ was found: Error rates were higher for the action overlap $(M=2.3 \%)$ than for the nooverlap $(M=1.2 \%)$ condition. The PRC obtained for error rates indicates that the Event B RT interpretations above are not due to a speed-accuracy trade-off. See Fig. 4.

\section{Event A feature frequency}

Because all low-frequency conjunctions were collapsed together and compared to the high-frequency conjunction, the sizes of the PRCs for the individual low-frequency conjunction relative to the high-frequency conjunction could not be compared. To determine whether the size of the PRC obtained for the high-frequency conjunction was larger than that for each of the low-frequency conjunctions, the feature conjunctions mapped to Event A were parsed into four different high-frequency feature conditions: (1) conjunction [a specific response hand (left or right) and movement direction (up or down) appeared together with high frequency-e.g., left-up], (2) hand [the response hand (left or right) was the same as for the high-frequency conjunction, and hence "hand" was a high-frequency feature, but the movement direction (up or down) differed from the highfrequency conjunction, and hence "movement" was a lowfrequency feature-e.g., left-down], (3) movement [the movement direction (up or down) was the same as for the high-frequency conjunction, and hence "movement" was a high-frequency feature, but the response hand (right or left) differed from the high-frequency conjunction, and hence "hand" was a low-frequency feature-e.g., right-up], and (4) none [none of the features (hand or movement direction) were the same as those for the high-frequency conjunction, 
and hence both "hand" and "movement" were lowfrequency features-e.g., right-down]. These four different conditions are represented in the top panel of Fig. 1.

A $2 \times 4 \times 4$ repeated measures ANOVA with the factors Action Overlap (overlap or no overlap), High-Frequency Feature (conjunction, hand, movement, or none), and Block Group (early, mid-early, mid-late, or late) was conducted separately on Event A percentages of errors, Event B correct RTs, and Event B percentages of errors. The correct-RT and error analyses for Event B were restricted to trials in which responses to Event A were correct. Only significant effects that involved the factor High-Frequency Feature are discussed. Figure 5 shows the mean correct RTs and percentages of errors for Event B as a function of action overlap and high-frequency feature (collapsed over block groups). As is evident in Fig. 5, the magnitudes of the PRC were equivalent between the high-frequency conjunction and the high-frequency "hand" feature conditions. Event A percentages of errors, which showed no significant effects of highfrequency feature, are presented in Table 1.

Event $B$ RTs There was a significant interaction between action overlap and high-frequency feature $[F(3,108)=$ $\left.4.44, p=.005, \eta_{\mathrm{p}}{ }^{2}=.11\right]$. See Fig. 5. Post hoc comparisons (Tukey's HSD) showed that RTs were slower for the action overlap than for the no-overlap condition (i.e., a PRC was
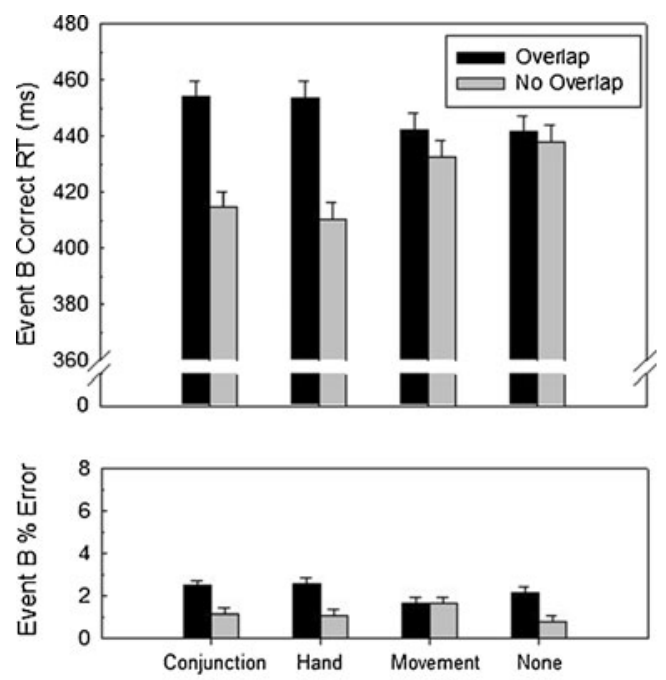

High-Frequency Feature for Event A

Fig. 5 Experiment 1: Event B correct RTs (upper panel) and percentages of errors (lower panel) for action overlap (overlap and no overlap) and each of the high-frequency feature conditions assigned to Event A (Conjunction = "hand" and "movement" features occurred together with high frequency, e.g., left-up; Hand = the high-frequency "hand" feature was paired with the low-frequency "movement" feature, e.g., left-down; Movement = the high-frequency "movement" feature was paired with the low-frequency "hand" feature, e.g., right-up; None $=$ the low-frequency "hand" feature and low-frequency "movement" feature were paired together, e.g., right-down). Each error bar represents one within-subjects standard error of the mean found) when Event A was the high-frequency conjunction (e.g., left-up; $M=40 \mathrm{~ms}$ slower, $p=.002$ ) and contained the high-frequency "hand" feature (e.g., left-down; $M=43 \mathrm{~ms}$ slower, $p<.001)$. Also, the sizes of the PRC were similar $(p>.95)$ for these two conditions. No RT differences were found between the action overlap and no-overlap conditions when Event A contained the high-frequency "movement" or no high-frequency features ( $p$ s $>.95)$. This indicates that the high-frequency "hand" feature code ("left" or "right") alone led to a significant PRC when the same high-frequency "hand" feature ("left" or "right") was mapped to Event B.

Event $B$ accuracy There were no significant effects found for high-frequency feature ( $p s>.07)$, suggesting that the RT interpretations above are not due to a speed-accuracy trade-off. See Fig. 5.

\section{Summary}

Unexpectedly, the sizes of the PRC were similar when Event A was the high-frequency conjunction or a low-frequency "movement" feature in conjunction with the high-frequency "hand" feature code ("left" or "right")— suggesting that the binding strengths were similar for these two conditions. This result alone does not compromise the idea that binding is a preliminary step in forming a more durable memory trace. However, this result is not consistent with the TEC, which predicts that binding strength among action features maintained in working memory (Event A) should be greater when a specific conjunction of action features (representing both the response hand and the movement direction) is presented with high as compared to low frequency. This result indicates that the binding strengths among Event A action features were equivalent for high- and low-frequency conjunctions when the first feature code (i.e., "left" or "right," representing hand) in the "hand + movement" action plan occurred with high frequency. Also, because no significant PRC was found for the low-frequency conjunctions that contained the high-frequency "movement" feature code ("up" or "down") or contained no high-frequency feature codes, this suggests that either binding was relatively weak for these conditions or that the "hand" action code for Event $\mathrm{B}$ was less effective in backward priming (reactivating) these bound action codes (and, hence, less likely to produce code confusion).

Although these findings suggest that the binding strengths were equivalent for the two conjunctions that contained the high-frequency "hand" feature code (the high-frequency conjunction and high-frequency "hand" conditions), it is also possible that the binding strengths were equivalent among all of the conjunction conditions, regardless of conjunction or feature frequency. That is, the size of the PRCs obtained could mainly reflect the 
Table 1 Event A percentages of errors for action overlap by high-frequency feature (conjunction, hand, movement, or none); within-subjects $S E \mathrm{~s}=1.89$ for Exp. 1 and 1.58 for Exp. 2

Action Overlap

\begin{tabular}{|c|c|c|c|c|c|c|c|c|}
\hline \multirow[b]{2}{*}{ Experiment } & \multicolumn{4}{|c|}{ Overlap } & \multicolumn{4}{|c|}{ No Overlap } \\
\hline & Conjunction & Hand & Movement & None & Conjunction & Hand & Movement & None \\
\hline 1 & 8.9 & 7.9 & 11.1 & 9.3 & 10.9 & 9.4 & 9.0 & 8.8 \\
\hline 2 & 10.7 & 10.8 & 9.4 & 10.0 & 11.1 & 12.7 & 14.0 & 13.7 \\
\hline
\end{tabular}

differences in the ability of Event B to backward prime the bound action codes represented by Event A. According to Hommel (2005), once features are bound (e.g., "left-up"), encountering one of these features again (e.g., "left") will reactivate its code, as well as the code(s) that it is bound with (e.g., "up"). As a result, confusion as to which code(s) correspond to the new event (e.g., "left" or "left-up") can occur, leading to a PRC (see the introduction and Fig. 1). Thus, PRCs are contingent on feature binding and on reactivation of this bound representation maintained in working memory. The ability of Event B to reactivate Event A should depend on the activation strength of the overlapping feature code (e.g., a "left" or "right" code, corresponding to the response hand), and activation strength should be greater for a high-frequency feature code than for a low-frequency feature code. Consistent with this interpretation, the size of the PRC was larger when the event maintained in memory (Event A) and the new action event (Event B) each contained the same high-frequency feature code (e.g., "left" for a lefthand response), as opposed to the same low-frequency feature code (e.g., "right" for a right-hand response). Thus, the action feature codes for Event A may have been bound together with equivalent strength, regardless of our frequency manipulation, but the probability of reactivating a previously planned action was increased when both the action events (Event A and Event B) contained the same high-frequency feature code.

Another possible interpretation is that binding strength was greater when the first feature code in the action sequence "hand + movement direction" occurred with high rather than with low frequency. It could be that feature binding proceeds in a forward direction, such that a highfrequency feature only produces increased binding strength if it occurs as the first feature in the action sequence. For example, in the action sequence "left hand move up," the binding strength may be greater when the high-frequency feature code corresponds to the response hand (e.g., "left") as opposed to the movement direction (e.g., "up"). The significant PRC found only when the "hand" code was high in frequency lends support to this possibility. Also, the failure to find a larger PRC in the high-frequency "movement" as compared to the no-high-frequency-feature condition suggests that having a high-frequency feature code occur toward the end of the action sequence did not increase binding strength. Recall that the order of action features for Event A ("hand" followed by "movement direction") is consistent with hierarchical models of action planning (e.g., Rosenbaum et al., 1984), research by Miller (1982), and participant self-report.

Although we failed to obtain evidence that binding strength was greater for the high-frequency conjunction than for the high-frequency "hand" condition, it is possible that the binding process might begin/occur earlier for the highfrequency conjunction. According to Colzato, Raffone, and Hommel (2006), if binding and associative learning go hand in hand (e.g., Miltner et al., 1999), then learning a feature conjunction should affect the way in which these features are bound in terms of strength and the speed of feature binding. To obtain a better understanding of whether conjunction frequency or the frequency of the first feature code in the action sequence influences binding, Experiment 2 was conducted.

\section{Experiment 2}

In Experiment 2, we determined whether increased conjunction frequency could cause action features to bind together earlier. To test this, the method was the same as in Experiment 1, except that Event A and Event B occurred over a short interval (150-ms SOA), so that presumably feature binding of Event A was not likely to precede the onset of Event B (e.g., Hommel \& Colzato, 2004). According to the TEC, if features are not yet bound together, they should be more available to prime future actions (i.e., actions associated with the new Event B), whereas features that are in the early process of feature binding should be less available to prime future actions. Thus, if features are bound together earlier for a high-frequency conjunction than for a lowfrequency conjunction, priming (facilitation) of the Event B response when Event A and B partly overlap should be greater when Event $\mathrm{A}$ is a low-rather than a high-frequency conjunction (at these short event SOAs). Hommel and colleagues estimated that feature binding for perceptual 
features begins approximately 250 to $500 \mathrm{~ms}$ after event onset (Hommel, 2002; Hommel \& Colzato, 2004). Consistent with this estimate, Hommel and Colzato (2004) found facilitation as opposed to a cost for visual events that partially overlapped when the SOA between events was $200 \mathrm{~ms}$.

Due to the short SOA between Events A and B, it was predicted that partial-repetition facilitation (PRF; faster responses to Event B) would occur in early blocks of trials, regardless of the high-frequency features assigned to Event A (conjunction, hand, movement, or none). Also, if binding occurred earlier for the high-frequency conjunction, then either a significant reduction in PRF or a PRC should be observed in the later blocks of trials - or at least in earlier block groups, as compared to the other high-frequency feature conditions (hand, movement, or none)—when Event $\mathrm{A}$ is a low-frequency conjunction. Finally, if an increase in conjunction frequency (or feature frequency) does not cause binding to occur earlier than 150-200 ms, a PRF should occur across all block groups to an equivalent degree, regardless of the high-frequency feature conditions corresponding to Event A.

\section{Method}

\section{Participants}

A group of 55 undergraduates participated. Experiment approval and the participant characteristics and compensation were the same as for Experiment 1. Fifteen of the participants were excluded: four for failing the $80 \%$-accuracy criterion in the practice block, seven for low Event A accuracy (less than $80 \%$ ), and four for moving while planning the response to Event A. The data were analyzed for 40 participants.

\section{Apparatus, stimuli, and procedure}

The apparatus, stimuli, and procedure were the same as in Experiment 1, except that the duration of Event A was $50 \mathrm{~ms}$, the duration of Event B was $50 \mathrm{~ms}$, and the duration of the screen that occurred between Events A and $\mathrm{B}$ was $100 \mathrm{~ms}$. Thus, the total duration between the onset of Event A and the disappearance of Event B was $200 \mathrm{~ms}$ (see Fig. 3).

Results and discussion

A $2 \times 4 \times 4$ repeated measures ANOVA with the factors Action Overlap (overlap or no overlap), High-Frequency Feature (conjunction, hand, movement, or none), and Block Group (early, mid-early, mid-late, or late) was conducted separately on Event A percentages of errors, Event B correct
RTs, and Event B percentages of errors. The mean correct RTs and percentages of errors for Event B were restricted to trials in which responses to Event A were correct. Figure 6 shows the mean correct RTs and percentages of errors for Event B as a function of action overlap and high-frequency feature, collapsed over block groups. As is shown in Fig. 6, no PRCs were found. However, the PRFs were smaller (suggesting that the binding process began earlier) in the high-frequency conjunction and high-frequency "hand" conditions than in the high-frequency "movement" and nohigh-frequency-feature conditions.

\section{Event A accuracy}

The average error rate for Event A was $11.6 \%$. We found a significant main effect of block group $[F(3,117)=$ $\left.19.98, p<.001, \eta_{\mathrm{p}}{ }^{2}=.34\right]$, indicating that accuracy improved across blocks (consistent with a practice effect). There was also a significant main effect of action overlap $[F(1,39)=$ $\left.12.24, p=.001, \eta_{\mathrm{p}}{ }^{2}=.24\right]$ and a significant interaction between action overlap and high-frequency feature $[F(3$, $\left.117)=2.90, p<.04, \eta_{\mathrm{p}}{ }^{2}=.07\right]$. See Table 1. Planned comparisons showed that recall was more accurate for the action overlap than for the no-overlap condition when Event A contained either the high-frequency "movement" feature or
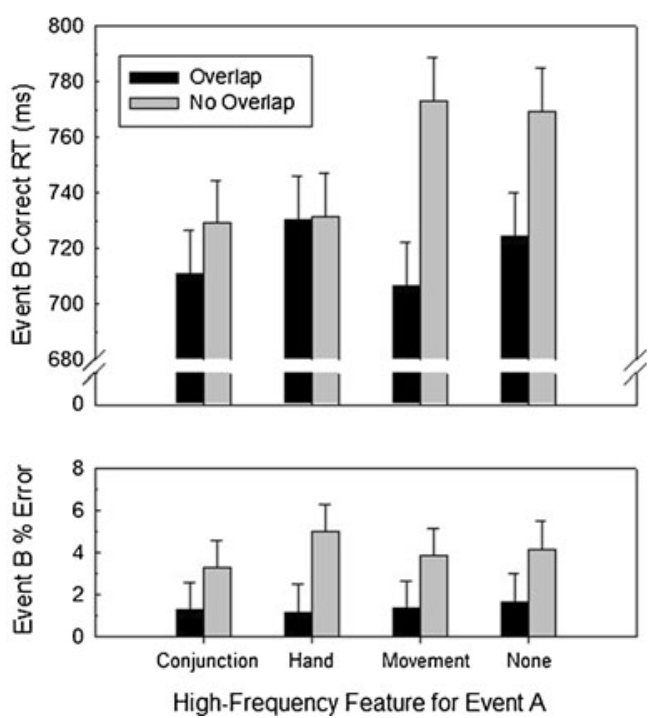

Fig. 6 Experiment 2: Event B correct RTs (upper panel) and percentages of errors (lower panel) for action overlap (overlap and no overlap) and each of the high-frequency feature conditions assigned to Event A (Conjunction = "hand" and "movement" features occurred together with high frequency, e.g., left-up; Hand = the high-frequency "hand" feature was paired with the low-frequency "movement" feature, e.g., left-down; Movement = the high-frequency "movement" feature was paired with the low-frequency "hand" feature, e.g., right-up; None = the low-frequency "hand" and "movement" features were paired together, e.g., right-down). Each error bar represents one within-subjects standard error of the mean 
no high-frequency features $(p \mathrm{~s}<.05)$, but not when Event A was the high-frequency conjunction or contained the highfrequency "hand" feature ( $p$ s $>.60)$. Thus, action overlap facilitated recall of Event A only when the overlapping "hand" feature ("left" or "right") was low in frequency.

\section{Event B RTs}

Significant main effects of block group $[F(3,117)=$ 47.47, $\left.p<.001, \eta_{\mathrm{p}}{ }^{2}=.55\right]$, high-frequency feature $[F$ $\left.(3,117)=7.59, p<.001, \eta_{\mathrm{p}}{ }^{2}=.16\right]$, and action overlap $\left[F(1,39)=27.22, p<.001, \eta_{\mathrm{p}}^{2}=.41\right]$ were found. Also, we found significant two-way interactions between action overlap and block group $[F(3,117)=4.514, p=.005$, $\left.\eta_{\mathrm{p}}{ }^{2}=.10\right]$ and between action overlap and high-frequency feature $\left[F(3,117)=6.70, p<.001, \eta_{\mathrm{p}}{ }^{2}=.15\right]$; the three-way interaction was not significant $[F(9,351)=$ $\left.1.09, p=.37, \eta_{\mathrm{p}}^{2}=.03\right]$. Planned comparisons conducted on the interaction between action overlap and block group showed that RTs were significantly faster for the action overlap than for the no-overlap condition in the mid-early, mid-late, and late block groups $(p \mathrm{~s}<.001)$, but not in the early block group $(p>.17)$. Thus, a PRF occurred across all blocks except for the early block of trials, which trended toward facilitation.

More importantly, planned comparisons conducted on the interaction between action overlap and highfrequency feature showed that RTs were faster for the action overlap than for the no-overlap condition when Event A contained the high-frequency "movement" feature $(p<.001)$ or no high-frequency features $(p<.001)$, but not when Event A was the high-frequency conjunction $(p>.10)$ or contained the high-frequency "hand" feature $(p>.90)$. Thus, a significant PRF was found only when Events A and B shared the low-frequency "hand" feature. Also, planned comparisons conducted on the PRF obtained (no action overlap RT - action overlap RT) for each of the high-frequency feature conditions showed that the PRF was significantly reduced when Event A was the high-frequency conjunction or contained the high-frequency "hand" feature, as compared to when Event A contained the high-frequency "movement" feature or no high-frequency features, $p<.001$. These findings indicate that when Events $\mathrm{A}$ and $\mathrm{B}$ partly overlapped, Event A was less effective in priming the response to Event B when Event A contained the high- versus the low-frequency "hand" feature. In accordance with the TEC, the events that contained the high-frequency "hand" feature were in the early process of feature binding, rendering them less available to prime the actions associated with the new event (Event B), whereas the events that contained the low-frequency "hand" feature were not yet bound together, and hence were more available, allowing them to prime the actions associated with Event B. ${ }^{2}$

Furthermore, the sizes of the PRF were similar when Event A contained the high-frequency "movement" feature $(M=66.8 \mathrm{~ms})$ and when it contained no high-frequency features $(M=44.9 \mathrm{~ms} ; p>.10)$. This suggests that when the low-frequency "hand" feature in these two conditions overlapped with the Event $\mathrm{B}$ response, they were equally able to prime the Event B response. Because priming by the lowfrequency "hand" feature was not reduced when paired with a high-frequency, as opposed to a low-frequency, "movement" feature, this indicates that the "hand" feature was equally available to prime its response in both of these feature frequency conditions. Thus, there was no evidence that binding occurred earlier when the low-frequency "hand" feature was paired with the high-frequency as opposed to the low-frequency "movement" feature. This finding, along with the finding that the PRF (and, hence, facilitative priming) was significantly reduced when "hand" was the high-frequency feature suggests that the binding process began earlier when the high-frequency feature occurred at the beginning rather than the end of the "hand + movement" action sequence.

\section{Event B accuracy}

The effects of block group $[F(3,117)=4.91, p=.003$, $\left.\eta_{\mathrm{p}}^{2}=.11\right]$ and action overlap $[F(1,39)=19.114, p<$ $\left..001, \eta_{\mathrm{p}}^{2}=.33\right]$ and the interaction between these two factors $\left[F(3,117)=2.97, p=.035, \eta_{\mathrm{p}}^{2}=.07\right]$ were significant. Planned comparisons showed that accuracy was greater in the action overlap than in the no-overlap condition in all block groups $(p s<.001)$ except the early block group. Also, accuracy for the action overlap condition was consistent across blocks $(p s>.95)$, while accuracy for the no-overlap condition decreased in the

\footnotetext{
${ }^{2}$ Note also that in Fig. 6 that response competition likely occurred in the no-action-overlap conditions because the first and second events had different response mappings on the same task dimension-for instance, left- and right-hand responses, respectively. Importantly, this response competition (greater RTs and errors for the no-overlap vs. overlap conditions) was larger when the "hand" feature was a lowfrequency feature (i.e., the high-frequency "movement" feature and nohigh-frequency-feature conditions) than when it was a high-frequency feature (i.e., the high-frequency conjunction and high-frequency "hand" conditions). This suggests that the action code associated with the high-frequency "hand" feature (e.g., "left") was less available to compete with the action code associated with the second event (e.g., "right" for a right-hand response) due to its participation in feature binding (see Mattson \& Fournier, 2008). This is further evidence that response priming (including that leading to response competition) was reduced when the "hand" feature (e.g., "left") was the high-frequency feature. Hence, binding likely occurred earlier when the first event contained a high- rather than a low-frequency feature on the response dimension (i.e., "hand") relevant to the second event.
} 
mid-late and final blocks relative to the mid-early block group $(p s<.01)$. Thus, a PRF for accuracy occurred in all high-frequency feature conditions, and the size of the PRF obtained increased across blocks. These findings are consistent with the Event B RT interpretations above and do not indicate a speed-accuracy trade-off.

\section{Summary}

The SOA between the two events (A and B) was shortened in order to determine the onset of the feature-binding process. Although no PRCs were observed, there was evidence that the binding process began earlier for events containing a high-frequency feature code at the beginning of the "hand + movement" action sequence. PRFs were similarly reduced when Event A contained a highfrequency "hand" feature code (i.e., the high-frequency conjunction and "hand" conditions) as compared to when it contained a low-frequency "hand" feature code (i.e., the high-frequency "movement" and no-high-frequency-feature conditions). Also, the sizes of the PRF found in the latter two cases were similar. If binding was contingent on an event containing a conjunction of high-frequency features, a reduction in PRF (or evidence of a PRC) should have been larger for the high-frequency conjunction than for all of the other high-frequency feature conditions. Also, if binding was dependent on the occurrence of a high-frequency feature alone (independent of where the high-frequency feature occurred in the action sequence), we should have obtained a smaller PRF when Event A contained a high-frequency "movement" feature than when it contained no highfrequency features. Our data show instead that the process of feature binding was determined by the frequency of the "hand" feature code ("left" or "right"), which was represented (and maintained in memory) as the first feature in the action sequence. Converging evidence that binding may be influenced by the first feature in the action sequence is presented in the General Discussion.

These findings are consistent with those of Experiment 1, and also help clarify the findings in that experiment. The low-frequency "hand" feature code was more effective than the high-frequency code in priming the response to the second event in Experiment 2. Therefore, the Experiment 1 results cannot be completely due to differences in the abilities of the high- and low-frequency "hand" features in Event B (the second event) to backward prime the action associated with Event A (the first event). Also, the fact that the low-frequency "hand" feature associated with the first event in Experiment 2 led to more facilitative priming than did the high-frequency "hand" feature indicates that binding was at least beginning to take place earlier (or at least occurred on a higher proportion of trials) when the first event contained a high-frequency feature at the beginning of the action sequence. The evidence in Experiment 2 that the binding process began earlier for the first event when it contained a high-frequency feature at the beginning of the response sequence suggests that the larger PRCs obtained in Experiment 1 for these conditions were partly due to the binding process occurring earlier for these conditions. This, in turn, could increase the overall strength of binding if the binding process requires time. However, the interval of $3.25 \mathrm{~s}$ between the first and second events in Experiment 1 should have been sufficient for binding to occur (although the estimates of binding [250-500 ms] are based on perceptual features, as opposed to action features); hence, it is likely that the increased frequency of a feature occurring at the beginning of the action sequence may have a greater influence on the overall strength of feature binding than on the onset of binding.

\section{General discussion}

In this study, we investigated whether temporary binding among feature codes relevant to an action event can be a preliminary step in creating a more durable memory trace of this action event. Consistent with this assumption, this study provides evidence that binding strength among action feature codes can increase when the specific action event (action feature conjunction) occurs with greater frequency. Moreover, we suggest that increasing the frequency of the first-specified feature in the action sequence alone can increase the strength and/or speed of action feature binding. An increase in the size of partial-repetition costs (Exp. 1) and a reduction in partial-repetition facilitation (Exp. 2) were found for the high-frequency conjunction (e.g., "left hand move up") and the low-frequency conjunction with the high-frequency "hand" feature (e.g., "left hand move down") as compared to the low-frequency conjunctions with the high-frequency "movement" feature (e.g., "right hand move up") and with no high-frequency features (e.g., "right hand move down"). Furthermore, the sizes of the partialrepetition costs and the reduction in partial-repetition facilitation were similar for the high-frequency conjunction and the low-frequency conjunction with the high-frequency "hand" feature (e.g., "left"). These findings suggest that both the high-frequency conjunction and the highfrequency "hand" condition showed greater and/or earlier binding strength than did the high-frequency "movement" and no-high-frequency-feature conditions. These findings, together with past research showing that the feature code ("left" or "right") representing hand should be located at the beginning of the action sequence (Miller, 1982; Rosenbaum, 1980), suggest that feature binding is contingent on the frequency of the first feature code in the action sequence. That is, increasing the frequency of the first-specified 
feature code in the action sequence (e.g., "left" or "right," corresponding to the action hand) is sufficient to increase binding among action feature codes.

This latter finding is inconsistent with one of the assumptions of the TEC. According to the TEC, the activation level of individual feature codes determines which feature codes within an event become bound together to form an action plan (or event file). The higher the activation strength of the feature code(s), the more likely they will participate in binding. It is assumed that feature codes that are task relevant will have higher activation strength than those that are not task relevant, and hence binding is more likely to occur among task-relevant features. The theory also assumes that the higher the activation strength of the task-relevant feature code(s), the greater the binding strength among those codes (Colzato, Raffone, \& Hommel, 2006; Colzato, Warrens, \& Hommel, 2006; Hommel et al., 2001). Thus, the theory predicts that increasing feature frequency, known to increase feature activation, should increase the binding strength among action features of the same event, particularly when the same action features are paired together with high frequency (Colzato, Raffone, \& Hommel, 2006; Colzato, Warrens, \& Hommel, 2006). In contrast to this prediction, in the present study we found no evidence that action features corresponding to the high-frequency event (conjunction) were bound together more strongly or earlier than those for a lowfrequency event that contained the high-frequency feature at the beginning of the action sequence. Evidence that the binding process is stronger and/or occurs earlier (or with higher probability) for events that contain a high-frequency feature at the beginning rather than at the end of the action sequence suggests that feature binding, at least among action features, is influenced by the order of action features in the action sequence.

For many action events, the order of action features must be preserved in an action plan (e.g., Jeannerod, 1997). For example, to put your car in first gear with a manual transmission, you must move the lever "left then up," not "up then left" - the latter response would get you nowhere and result in a most unpleasant sound! As a result, it is possible that a high-frequency feature that occurs at the beginning of the action sequence (e.g., "move left") may influence the strength and/or the rate at which this feature binds to an action feature that occurs later in the action sequence. This would also mean that the frequency of the action feature that occurs late in the constructed action sequence (e.g., "move up") may have little influence on the strength or rate at which this feature binds to an action feature that occurs at the beginning of the action sequence. That is, action feature binding may work in a serial manner, binding features forward in the order that they are to be executed. As such, the activation of the first feature may determine the binding time as well as the strength between the two task-relevant features. This can differ from feature binding for perceptual features, which may bind features together in a parallel fashion within the focus of attention (Treisman, 1996, 1998; Treisman \& Gelade, 1980), unless the goal is to keep track of a temporal sequence of perceptual features. When the goal is to keep track of a temporal sequence of perceptual features, research shows a benefit in accurately recalling the first item in a memorized sequence, with recall accuracy and speed progressively declining toward the end of the memorized sequence. This suggests that people tend to access the item at the beginning of the sequence and to search forward for the target (e.g., referred to as "anchoring"; Klahr, Chase, \& Lovelace, 1983; Sternberg, 1969). It also suggests that items can be associated in a way that preserves temporal order, which is particularly important when talking, singing a song, or executing a manual motor action (see also linearization models proposed for sentence production, e.g., Hartsuiker \& Westenberg, 2000). Thus, it is very possible that feature binding of action sequence information could have a forward-activating bias.

Consistent with the present findings and the forwardbinding interpretation, the partial-repetition costs found between action events in all other studies to our knowledge have occurred when action overlap (or action and perceptual feature overlap) between the two events involved the first action feature in the sequence (according to the task instructions) maintained in memory. Also, the results from a recent study conducted in our lab are consistent with the interpretation that overlap involving the first action feature in the action sequence predicts partial-repetition costs (Fournier, Gallimore, \& Feiszli, submitted). Participants planned a two-movement joystick response to the first event (e.g., left-up or up-left) with their dominant hand and maintained this action plan in memory while executing a quick joystick movement to the second event (e.g., to the left) with their dominant hand. Partial-repetition costs occurred when executing the response to the second event (e.g., left), but only when the action for the second event overlapped with the first feature of the action sequence maintained in memory (e.g., left-up), not with the second feature (e.g., up-left). This finding suggests that the second event was able to reactivate (backward prime) the action plan maintained in working memory only in a forward direction. For example, when planning the action "move lever left then up," the features "left-up" become associated with each other in a specific order (i.e., execute "left then up," not "up then left"). As a consequence, when the second event requires the first action feature "left," the second action feature "up" is also activated (forward priming); however, if the second event requires the latter action feature "up," the earlier action feature "left" will not become activated. Because the action plan maintained in working memory may only be reactivated (primed) in a forward direction, it is possible 
that feature binding among action features occurs only in a forward direction, as opposed to both a forward and backward direction.

This conclusion fits well with physiological evidence showing that serial order is preserved when planning an action sequence, as well as physiological evidence suggesting that the representation of the first feature in the action sequence is critical in preserving this serial order. For example, Gerloff, Corwell, Chen, Hallett, and Cohen (1997) showed that high-frequency repetitive transcranial magnetic stimulation affecting the supplementary motor area (SMA) in humans interfered with the preparation and performance of future components of sequential finger movements rehearsed from memory, with participants reporting that they "did not know anymore what series to press next" (p. 1598). Also, Baldauf, Cui, and Andersen (2008) trained monkeys to reach to two different target locations in a particular order indicated by a cue, and they showed that some cells in the parietal reach region responded to both the first and second goals of a planned reaching sequence during a memory period, with cell activity biased toward the first goal. Furthermore, Tanji and Shima (1994) found cells in the SMA of the macaque that were preferentially active during the interval between two specific, learned sequential movements; these cells were active before a "pull" movement if the previous movement was "push," but not if the previous movement was "turn." These researchers suggested that order-specific cellular activity in the SMA could provide a signal about the order of forthcoming movements useful for programming the temporal structure of an intended motor act (performed on the basis of memory) that includes multiple movements. On the basis of this physiological evidence, it is very possible that feature binding of action sequence information could have a forward-activating bias. However, this possibility is not addressed by the TEC.

In conclusion, binding processes are thought to solve problems resulting from distributed processing, but the finding that feature frequency influenced feature-binding strength and time suggests that feature binding can also be a preliminary step in creating a more durable memory trace (Colzato, Raffone, \& Hommel, 2006; Hommel et al., 2001; Logan, 1988). According to the TEC, increased activation among action features (based on task relevance and frequency) should increase the probability as well as the onset and strength of feature bindings. Eventually, these actions are generated automatically and consist of feature codes integrated in long-term memory. Interestingly, however, we found no evidence that this process is contingent on the presentation frequency of task-relevant feature conjunctions; instead, the frequency of the leading feature in the action sequence of the task-relevant conjunction appears to be sufficient. Our findings provide an update to the TEC and suggest that action planning may involve serial binding of action features into an action plan in the order that the action features are to be executed. That is, the binding of action features results in associations among features that are not bidirectional, as is the case for perceptual features, for which temporal order is not goal relevant; instead, action features are associated in a forward direction - at least when temporal order is goal relevant. Our findings and interpretations are consistent with what might be predicted to occur on the basis of the hierarchical structure of action plans (e.g., Rosenbaum, 1980). However, a more systematic investigation of forward feature binding of action feature codes is needed and is currently underway in our laboratory.

\section{References}

Baldauf, D., Cui, H., \& Andersen, R. (2008). The posterior parietal cortex encodes in parallel both goals for double-reach sequences. The Journal of Neuroscience, 28, 10081-10089.

Chao, L. L., \& Martin, A. (2000). Representation of manipulable manmade objects in the dorsal stream. NeuroImage, 12, 478-484. doi:10.1006/nimg.2000.0635

Colzato, L., Raffone, A., \& Hommel, B. (2006). What do we learn from binding features? Evidence for Mulitlevel Feature Integration. Journal of Experimental Psychology: Human Perception and Performance, 32, 705-716.

Colzato, L., Warrens, M., \& Hommel, B. (2006). Priming and binding in and across perception and action: A correlational analysis of the internal structure of event files. The Quarterly Journal of Experimental Psychology, 59, 1785-1804.

Desimone, R., \& Duncan, J. (1995). Neural mechanisms of selective visual attention. Annual Review of Neuroscience, 18, 193-222. doi:10.1146/annurev.ne.18.030195.001205

Engel, A., \& Singer, W. (2001). Temporal binding and the neural correlates of sensory awareness. Trends in Cognitive Sciences, 5 , $16-25$.

Eriksen, B. A., \& Eriksen, C. W. (1974). Effects of noise letters upon the identification of a target letter in a nonsearch task. Perception \& Psychophysics, 16, 143-149. doi:10.3758/BF03203267

Forster, J., Liberman, N., \& Friedman, R. (2008). What do we prime? On distinguishing between semantic priming, procedural priming, and goal priming. In E. Morsella, J. A. Bargh, \& P. M. Gollwitzer (Eds.), Oxford handbook of human action (pp. 173-193). New York, NY: Oxford University Press.

Fournier, L. R., Wiediger, M. D., McMeans, R., Mattson, P. S., Kirkwood, J., \& Herzog, T. (2010). Holding a manual response sequence in memory can disrupt vocal responses that share semantic features with the manual response. Psychological Research, 74, 359-369.

Georgopoulos, A. (1990). Neurophysiology of reaching. In M. Jeannerod (Ed.), Attention and performance XIII: Motor representation and control (pp. 227-263). Hillsdale, NJ: Erlbaum.

Gerloff, C., Corwell, B., Chen, R., Hallett, M., \& Cohen, L. G. (1997). Stimulation over the human supplementary motor area interferes with the organization of future elements in complex motor sequences. Brain, 120, 1587-1602.

Grafton, S., Fadiga, L., Arbib, M., \& Rizzolatti, G. (1997). Premotor cortex activation during observation and naming of familiar tools. NeuroImage, 6, 231-236. 
Hartsuiker, R. J., \& Westenberg, C. (2000). Word order priming in written and spoken sentence production. Cognition, 75, B27-B39. doi:10.1016/S0010-0277(99)00080-3

Hommel, B. (2002). Responding to object files: Automatic integration of spatial information revealed by stimulus-response compatibility effects. The Quarterly Journal of Experimental Psychology, $55 A, 567-580$.

Hommel, B. (2004). Event files: Feature binding in and across perception and action. Trends in Cognitive Sciences, 8, 494-500. doi:10.1016/j.tics.2004.08.007

Hommel, B. (2005). Perception in action: Multiple roles of sensory information in action control. Cognitive Processing, 6, 3-14.

Hommel, B., \& Colzato, L. (2004). Visual attention and the temporal dynamics of feature integration. Visual Cognition, 11, 483-521.

Hommel, B., Müsseler, J., Aschersleben, G., \& Prinz, W. (2001). The Theory of Event Coding (TEC): A framework for perception and action planning. The Behavioral and Brain Sciences, 24, 849878. doi:10.1017/S0140525X01000103

Jeannerod, M. (1997). The cognitive neuroscience of action. Oxford, U.K.: Blackwell.

Kalaska, J. F., \& Hyde, M. L. (1985). Area 4 and area 5: Differences between load direction-dependent discharge variability of cells during active postural fixation. Experimental Brain Research, 59, 197-202.

Klahr, D., Chase, W. G., \& Lovelace, E. A. (1983). Structure and process in alphabetic retrieval. Journal of Experimental Psychology: Learning, Memory, and Cognition, 9, 462-477.

Logan, G. D. (1988). Toward an instance theory of automatization. Psychological Review, 95, 492-527. doi:10.1037/0033295X.95.4.492

Martin, A., Wiggs, C. L., Ungerleider, L. G., \& Haxby, J. V. (1996). Neural correlates of category-specific knowledge. Nature, 379, 649-652.

Mattson, P. S., \& Fournier, L. R. (2008). An action sequence held in memory can interfere with response selection of a target stimulus, but does not interfere with response activation of noise stimuli. Memory \& Cognition, 36, 1236-1247.

Miller, J. (1982). Discrete versus continuous stage models of human information processing: In search of partial output. Journal of Experimental Psychology: Human Perception and Performance, 8, 273-296.

Miltner, W. H. R., Braun, C., Arnold, M., Witte, H., \& Taub, E. (1999). Coherence of gamma-band EEG activity as a basis for associative learning. Nature, 397, 434-436.
Müsseler, J., \& Hommel, B. (1997). Blindness to response-compatible stimuli. Journal of Experimental Psychology: Human Perception and Performance, 23, 861-872. doi:10.1037/0096-1523.23.3.861

Riehle, A., \& Requin, J. (1989). Monkey primary motor and premotor cortex: Single-cell activity related to prior information about direction and extent of an intended movement. Journal of Neurophysiology, 61, 534-549.

Rosenbaum, D. A. (1980). Human movement initiation: Specification of arm, direction, and extent. Journal of Experimental Psychology: General, 109, 444-474.

Rosenbaum, D. A., Inhoff, A. W., \& Gordon, A. M. (1984). Choosing between movement sequences: A hierarchical editor model. Journal of Experimental Psychology: General, 113, 372-393.

Singer, W. (1994). Putative functions of temporal correlations in neocortical processing. In C. Koch \& J. L. Davis (Eds.), Large-scale neuronal theories of the brain (pp. 201-238). Cambridge, MA: MIT Press.

Sternberg, S. (1969). Memory scanning: Mental processes revealed by reaction time experiments. American Scientist, 57, 421-457.

Stoet, G., \& Hommel, B. (1999). Action planning and the temporal binding of response codes. Journal of Experimental Psychology: Human Perception and Performance, 25, 1625-1640.

Tanji, J., \& Shima, K. (1994). Role for supplementary motor area cells in planning several movements ahead. Nature, 371, 413-416. doi: $10.1038 / 371413 \mathrm{a} 0$

Terry, W. (2003). Learning and memory: Basic principles, processes, and procedures (2nd ed.). Boston, MA: Allyn \& Bacon.

Treisman, A. (1996). The binding problem. Current Opinion in Neurobiology, 6, 171-178.

Treisman, A. (1998). Feature binding, attention and object perception. Philosophical Transactions of the Royal Society B, 353, 1295-1306.

Treisman, A. M., \& Gelade, G. (1980). A feature-integration theory of attention. Cognitive Psychology, 12, 97-136. doi:10.1016/00100285(80)90005-5

Voss, J. (1972). On the relationship of associative and organizational processes. In E. Tulving \& W. Donaldson (Eds.), Organization of memory (pp. 167-194). New York, NY: Academic Press.

Wiediger, M. D., \& Fournier, L. R. (2008). An action sequence withheld in memory can delay execution of visually guided actions: The generalization of response compatibility interference. Journal of Experimental Psychology: Human Perception and Performance, 34, 1136-1149. doi:10.1037/00961523.34.5.1136

Wühr, P. (2006). The Simon effect in vocal responses. Acta Psychologica, 121, 210-226. doi:10.1016/j.actpsy.2004.12.003 